\title{
Treating patients with brain metastases has evolved: scalp-sparing, hippocampal avoidance whole brain radiotherapy with simultaneous integrated boost
}

\author{
Irfan Ahmad, Kundan Singh Chufal, Chandi Prasad Bhatt, Sandeep Rathour
}

Department of Radiation Oncology, Batra Hospital \& Medical Research Centre, New Delhi, India

\section{Correspondence to \\ Dr Irfan Ahmad,}

irfan.a@icloud.com

Accepted 27 November 2017

CrossMark

To cite: Ahmad I, Chufal KS, Bhatt CP, et al. BMJ Case Rep Published Online First: [please include Day Month Year]. doi:10.1136/bcr-2017 223449

\section{DESCRIPTION}

A 43-year-old male with a history of receiving treatment for squamous cell carcinoma (SqCC) of lung 2 years back presented with a complaint of a single episode of self-resolving generalised tonic-clonic seizure 1 day prior. General physical and neurological examinations were unremarkable. An MRI of the brain revealed a well-defined, enhancing, space-occupying lesion (SOL) in the right temporal lobe (figure 1). He was started on oral dexamethasone, oral phenytoin (after an intravenous loading dose) and underwent a whole body ${ }^{18}$ flourodeoxyglucose positron emission tomography CT $\left({ }^{18}\right.$ FDG PET-CT), which revealed increased FDG uptake in the SOL without evidence of metastatic disease elsewhere (figure 1). A diagnosis of oligometastatic SqCC lung (cTx, cNx and cM1b) was made, and the options for management were discussed with the patient.

He refused surgery (based on risk for postsurgical neurological deficit) and stereotactic

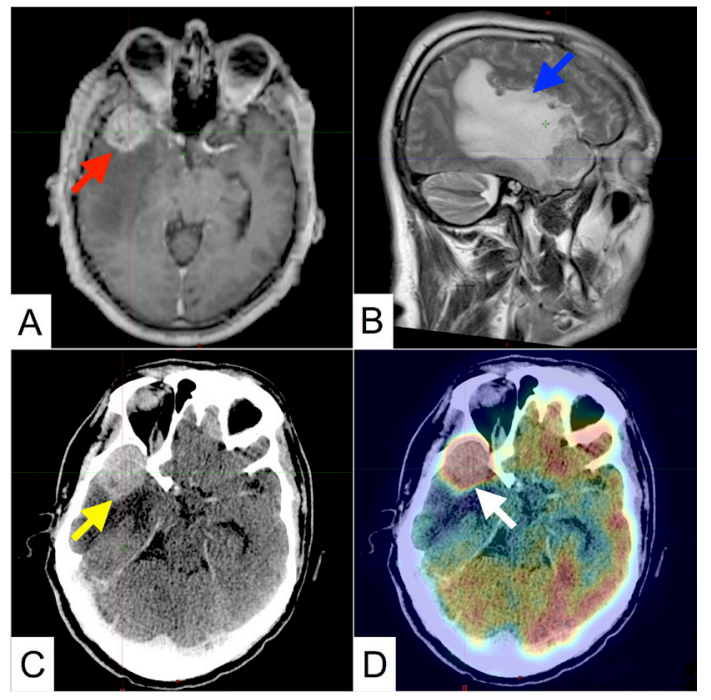

Figure 1 Pretreatment MRI and positron emission tomography CT (PET-CT) images. (A) T1-weighted contrast-enhanced axial image reveals a well-defined space occupying lesion in the right temporal lobe with intense contrast enhancement (red arrow), effacement of the right lateral ventricle and midline shift. (B) T2weighted sagittal image shows extensive perilesional oedema extending up to the parietal lobe (blue arrow). (C) Contrast-enhanced axial CT image shows the lesion (yellow arrow). (D) PET axial image fused with contrast enhanced CT showing FDG avidity within the lesion. Maximum standardised uptake value within the lesion was 20.5 (white arrow). radiosurgery (based on anticipated need for future retreatment for intracranial disease recurrence, the costs of which were unacceptable to him). The risk of neurocognitive decline and permanent alopecia with whole brain radiotherapy (WBRT) was also unacceptable. We planned and treated the patient with scalp-sparing, hippocampal avoidance WBRT (30 Gray in 10 fractions, 2 weeks) with simultaneous integrated boost (45 Gray in 10 fractions, 2 weeks) delivered by image-guided volumetric modulated arc therapy (figure 2). Treatment planning and delivery were performed on Varian Eclipse V.13.5 and Varian TrueBeam V.2.5, respectively (Varian Medical Systems, Palo Alto, California, USA). Daily image guidance was performed with pretreatment cone beam CT (CBCT) verification, intratreatment $\mathrm{kV}$ planar verification and post-treatment CBCT.

The patient developed transient alopecia 2 weeks post-treatment with full recovery at 2 month follow-up (figure 3), and neurocognitive function (assessed by Hopkins Verbal Learning Tool-Revised) also remained stable on follow-up compared with pretreatment levels. One year hence, the patient has achieved near-complete response intracranially and remains progression free elsewhere, without any systemic therapy (figure 3).

The standard management of a patient with solitary brain metastasis is either upfront surgery, stereotactic radiosurgery (SRS), WBRT or a combination of these modalities. ${ }^{1}$ While surgery is considered the standard of care, the rate of inhospital mortality is $2.3 \%$, and $17 \%$ are discharged to long-term care facilities or rehabilitation centres. ${ }^{1}$ In recent years, there is an increased recognition of the deleterious effects of WBRT on neurocognition, especially memory, which is not represented in the Mini-Mental Status Examination score. The key determinant of memory dysfunction after radiotherapy to the brain is the dose received by the hippocampus, a reservoir of neural stem cells responsible for the formation of new memories. $^{2}$ SRS is considered a viable alternative to surgery with excellent local control and has better neurocognitive outcomes compared with WBRT, at the cost of higher rate of intracranial disease progression, which requires retreatment, usually with SRS. ${ }^{1}$ Results from a recent trial suggest that sparing the hippocampus preserves neurocognitive function and quality of life in patients undergoing radiotherapy for brain metastases. ${ }^{2}$ Another trial recently demonstrated that WBRT 


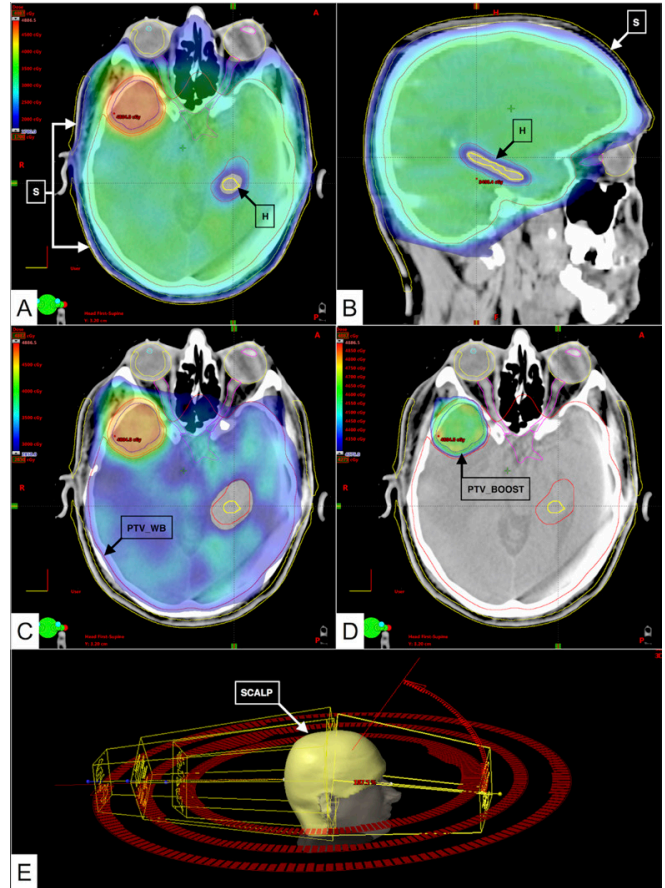

Figure 2 Treatment planning images. $(A, B)$ Dose colour wash in the axial and left parasagittal plane, with the lower limit set to $17 \mathrm{~Gy}$. The left hippocampus is shown with the black boxed arrow ' $\mathrm{H}$ ', and the scalp is shown with the white boxed arrow ' $S$ '. The maximum dose $\left(D_{\max }\right)$ allowed to the hippocampus as per RTOG 0933 protocol was $17 \mathrm{~Gy}$ and dose received by $100 \%\left(D_{100 \%}\right)$ of the hippocampal volume could not exceed $10 \mathrm{~Gy}^{2}$ In this patient, the $\mathrm{D}_{\text {max }}$ was $16.89 \mathrm{~Gy}$ and $D_{100 \%}$ was $9.84 \mathrm{~Gy}$, which was achieved by creating a planning organ at risk volume (PRV) of $5 \mathrm{~mm}$ around the hippocampus. The planning parameter for the scalp was set to the as low as reasonably achievable principle. The mean dose received by the scalp was $14.86 \mathrm{~Gy}$. (C) Dose colour wash in the axial plane with the lower limit set to $95 \%$ of $30 \mathrm{~Gy}$ to demonstrate adequate coverage of the whole brain planning target volume (PTV_WB), demonstrated by the black boxed arrow 'PTV_WB'. It was created by expanding the whole brain clinical target volume (CTV_WB) by $3 \mathrm{~mm}$, as per our institutional protocol for image-guided treatment delivery. This volume was subtracted from the hippocampal PRV for planning, and the optimisation parameter utilised was volume receiving $95 \%\left(\mathrm{~V}_{95 \%}\right)$ of the prescribed dose (30 Gy) should be more than $98 \%$. In this patient, the PTV_WB V colour wash in the axial plane with the lower limit set to $95 \%$ of $45 \mathrm{~Gy}$ to demonstrate adequate coverage of the boost planning target volume (PTV_Boost), demonstrated by the black boxed arrow 'PTV_Boost'. Also note the sharp dose fall medially to spare critical neurological structures. It was created by expanding the gross tumour volume (GTV) as seen on rigidly registered MRI images, by $3 \mathrm{~mm}$, as per our institutional protocol for image-guided treatment delivery. The optimisation parameter was volume receiving $95 \%\left(\mathrm{~V}_{95 \%}\right)$ of the prescribed dose (45 Gy) should be more than $98 \%$. In this patient, the PTV_Boost $\mathrm{V}_{95 \%}$ was $99.99 \%$. (E) Three-dimensional representation of the patient's scalp and the arc geometry utilised in creating this plan. All arcs (three coplanar and one non-coplanar) were planned using a single isocentric technique.

with a higher delivered dose to the metastasis is feasible with an overall response rate of $78 \% .^{3}$

In conclusion, modern radiotherapy techniques permit the customisation of treatment as per the patient's needs.

Twitter Follow Irfan Ahmad @irfanROres and Chandi Prasad Bhatt @cpbhatt

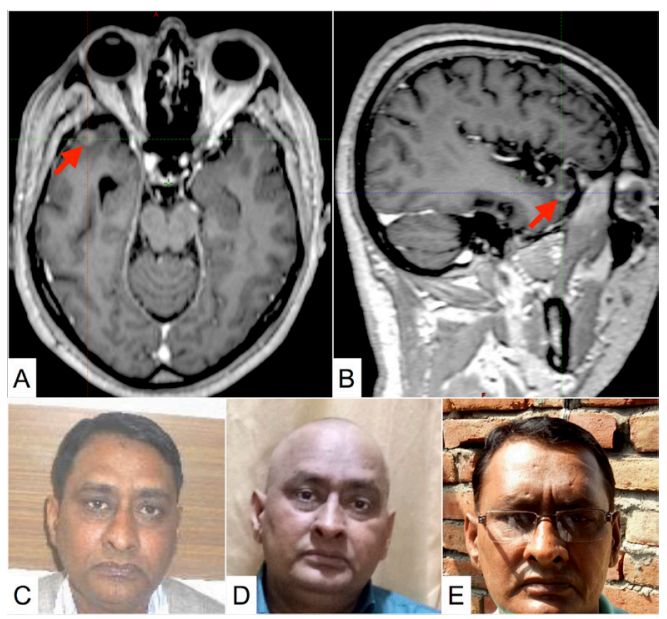

Figure 3 Post-treatment MRI and patient images. $(A, B)$ T1-weighted contrast enhanced axial and sagittal images reveal near complete response in the lesion(red arrows). (C) Pretreatment patient image demonstrating scalp hair distribution. (D) Post-treatment patient image taken 2 weeks after completion of treatment demonstrating alopecia totalis. (E) Post-treatment patient image taken 2 months after completion of treatment demonstrating return of normal scalp hair distribution.

\section{Learning points}

- Sparing the scalp and hippocampus is possible using modern radiotherapy techniques, without compromising therapeutic efficacy.

- Incorporating a simultaneous boost to large volume intracranial metastases induces long-term durable response.

- Sparing the scalp and hippocampus can counteract the issues of alopecia and memory dysfunction associated with whole brain radiotherapy.

Contributors IA is the treating junior consultant (radiotherapy), author of the paper, responsible for drafting the manuscript and revising it. He is the guarantor. KSC is the supervising treating consultant (radiotherapy) and participated in article formulation, editing and oversight. CPB is the medical physicist, responsible for generating the radiation treatment plan, performing quality assurance of delivered plan and also participated in article editing. SR is the radiation technologist, responsible for creating the treatment setup, ensuring reproducible setup and treatment delivery during the course of treatment. He also participated in article editing.

Competing interests None declared.

Patient consent Obtained.

Provenance and peer review Not commissioned; externally peer reviewed.

(c) BMJ Publishing Group Ltd (unless otherwise stated in the text of the article) 2017. All rights reserved. No commercial use is permitted unless otherwise expressly granted.

\section{REFERENCES}

Nichols EM, Patchell RA, Regine WF. et a/Palliation of brain and spinal cord metastases. In: Halperin EC, Brady LW, Wazer DE, Perez CA, . eds. Perez \& Brady's principles and practice of radiation oncology. 6th ed. Philadelphia: Lippincott Williams, 2013:1766-78.

2 Gondi V, Pugh SL, Tome WA, et al. Preservation of memory with conformal avoidance of the hippocampal neural stem-cell compartment during whole-brain radiotherapy for brain metastases (RTOG 0933): a phase II multi-institutional trial. J Clin Oncol 2014;32:3810-6.

3 Ferro M, Chiesa S, Macchia G, et al. Intensity Modulated Radiation Therapy With Simultaneous Integrated Boost in Patients With Brain Oligometastases: A Phase 1 Study (ISIDE-BM-1). Int J Radiat Oncol Biol Phys 2017;97:82-90. 
Copyright 2017 BMJ Publishing Group. All rights reserved. For permission to reuse any of this content visit http://group.bmj.com/group/rights-licensing/permissions.

BMJ Case Report Fellows may re-use this article for personal use and teaching without any further permission.

Become a Fellow of BMJ Case Reports today and you can:

- Submit as many cases as you like

- Enjoy fast sympathetic peer review and rapid publication of accepted articles

Access all the published articles

Re-use any of the published material for personal use and teaching without further permission

For information on Institutional Fellowships contact consortiasales@bmjgroup.com

Visit casereports.bmj.com for more articles like this and to become a Fellow 